\title{
Quantized thermal conductance of dielectric quantum wires
}

\author{
Luis G. C. Rego and George Kirczenow \\ Department of Physics, Simon Fraser University, Burnaby, B.C., Canada V5A $1 S 6$
}

(Received 10 December 1997)

\begin{abstract}
Using the Landauer formulation of transport theory, we predict that dielectric quantum wires should exhibit quantized thermal conductance at low temperatures in a ballistic phonon regime. The quantum of thermal conductance is universal, independent of the characteristics of the material, and equal to $\pi^{2} k_{B}^{2} T / 3 h$ where $k_{B}$ is the Boltzmann constant, $h$ is Planck's constant and $T$ is the temperature. Quantized thermal conductance should be experimentally observable in suspended nanostructures adiabatically coupled to reservoirs, devices that can be realized at the present time.
\end{abstract}

PACS: 85.30.Vw, 73.23.Ad, 63.22.+m

Typeset using REVTEX 
During the last two decades, the physics of electron transport in one dimension has attracted a great deal of attention. Some remarkable associated phenomena have been the quantum Hall effect discovered by von Klitzing, Dorda and Pepper [1] and the quantized conductance of ballistic point contacts discovered by van Wees et al. [2] and Wharam et al. [3] Here the signature of one-dimensional conduction has been the quantization of the twoterminal and Hall electrical conductances in multiples of the fundamental quantum $e^{2} / h$, as

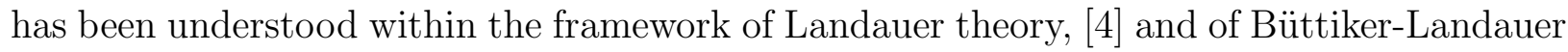
theory, [5] respectively.

One-dimensional phonon transport should also be possible. However, despite the long standing theoretical interest in this topic that goes back to the 1920's, [6] the question whether the phonon thermal conductance should be quantized in one dimension has to our knowledge not been addressed either theoretically or experimentally. Recent advances in nanotechnology have made experimental investigation of this question feasible; we note especially the detection of nanowire phonon subbands by Seyler and Wybourne [7] and the measurement of the thermal conductance of a suspended nanostructure by Tighe, Worlock and Roukes [8].

The purpose of this Letter is to demonstrate theoretically that in a low temperature regime dominated by ballistic massless phonon modes the phonon thermal conductance of a one dimensional quantum wire is quantized, the fundamental quantum of thermal conductance being $\pi^{2} k_{B}^{2} T / 3 h$, where $k_{B}$ is the Boltzmann constant, $h$ is Planck's constant and $T$ is the temperature. We also establish the conditions that should be met for the experimental observation of this novel phenomenon.

Our starting point is the Landauer energy flux

$$
\dot{Q}=\sum_{\alpha} \int_{0}^{\infty} \frac{d k}{2 \pi} \hbar \omega_{\alpha}(k) v_{\alpha}(k)\left(\eta_{R}-\eta_{L}\right) \zeta_{\alpha}(k)
$$

carried by a quantum wire connecting two reservoirs labeled $\mathrm{R}$ and L. Here $\omega_{\alpha}(k)$ and $v_{\alpha}(k)$ are the frequency and velocity of normal mode $\alpha$ of the quantum wire with wave-vector $k$, $\zeta_{\alpha}(k)$ is the phonon transmission probability through the wire and $\eta_{i}(\omega)=1 /\left(e^{h \omega / k_{B} T_{i}}-1\right)$ 
represents the thermal distribution of phonons in the reservoirs, assumed to be a Planck distribution at temperature $T_{i}$. The cross sectional area of the wire is assumed to be of the order of hundreds of $\mathrm{nm}^{2}$, so that the lateral confinement produces finite gaps in the dispersion relation of the phonon frequencies. Eq. (11) transforms to

$$
\dot{Q}=\frac{1}{2 \pi} \sum_{\alpha} \int_{\omega_{\alpha}(0)}^{\infty} d \omega \hbar \omega\left(\eta_{R}(\omega)-\eta_{L}(\omega)\right) \zeta_{\alpha}(\omega)
$$

since the phonon velocity $v_{\alpha}(k)=\partial \omega_{\alpha} / \partial k$ is canceled by the $1 \mathrm{D}$ density of states $g\left(\omega_{\alpha}\right)=$ $\partial k / \partial \omega_{\alpha}$.

The reservoir-to-reservoir thermal conductance of the wire is $\kappa=\dot{Q} / \Delta T$, where $\Delta T=$ $T_{R}-T_{L}$ is the temperature difference between the reservoirs. It follows that

$\kappa=\frac{1}{2 \pi}\left\{\sum_{\alpha}^{N_{\alpha}} \int_{0}^{\infty} d \omega \hbar \omega\left(\frac{\eta_{R}(\omega)-\eta_{L}(\omega)}{\Delta T}\right) \zeta_{\alpha}(\omega)+\sum_{\alpha^{\prime}}^{N_{\alpha^{\prime}}} \int_{\omega_{\alpha^{\prime}}(0)}^{\infty} d \omega \hbar \omega\left(\frac{\eta_{R}(\omega)-\eta_{L}(\omega)}{\Delta T}\right) \zeta_{\alpha^{\prime}}(\omega)\right\}$.

We have separated Eq.(3) in two parts, so that the first term represents the conductance of the massless modes with $\omega_{\alpha}(0)=0$ and the second is the contribution to the thermal conductance due to the higher energy modes, which have a finite cut-off frequency $\omega_{\alpha^{\prime}}(0) \neq 0$.

For the moment let us assume perfectly adiabatic contact between the thermal reservoirs and the ballistic quantum wire, so that $\zeta(\omega)=1$. For this idealized case integration of Eq.(3) yields

$\kappa=\frac{k_{B}^{2} \pi^{2}}{3 h}\left(\frac{T_{R}+T_{L}}{2}\right) N_{\alpha}+\frac{k_{B}^{2}}{h} \sum_{\alpha^{\prime}}^{N_{\alpha^{\prime}}}\left\{\frac{\pi^{2}}{3}\left(\frac{T_{R}+T_{L}}{2}\right)+\frac{1}{\Delta T}\left[T_{R}^{2} \operatorname{dilog}\left(e^{x_{R \alpha^{\prime}}}\right)-T_{L}^{2} \operatorname{dilog}\left(e^{x_{L \alpha^{\prime}}}\right)\right]\right\}$,

where $x_{(R, L) \alpha^{\prime}}=\hbar \omega_{\alpha^{\prime}}(0) / k_{B} T_{(R, L)}$, for the modes with finite cut-off frequencies.

Equation (1) predicts a remarkable behavior for the thermal transport properties of 1D phonon systems: Each massless mode presents a universal thermal conductance equal to the product of the averaged reservoir temperature and the universal constant $k_{B}^{2} \pi^{2} / 3 h$. The higher energy modes, on the other hand, show a dependence on the intrinsic properties of the material and on the geometrical parameters of the sample through the cut-off frequencies $\omega_{\alpha^{\prime}}(0)$. However their contribution to $\kappa$ is exponentially small at low temperatures. In the limit $\Delta T \rightarrow 0$ we find 


$$
\kappa=\frac{k_{B}^{2} \pi^{2}}{3 h} T N_{\alpha}+\frac{k_{B}^{2}}{h} T \sum_{\alpha^{\prime}}^{N_{\alpha^{\prime}}}\left\{\frac{\pi^{2}}{3}+f\left(x_{0}\right)+\frac{x_{0}^{2} e^{x_{0}}}{e^{x_{0}}-1}\right\}
$$

with $f(x)=2 \operatorname{dilog}\left(e^{x}\right)$ and $x_{0}=\hbar \omega_{\alpha^{\prime}}(0) / k_{B} T$.

Expressions (4) and (5) represent an idealized case in which the transmission of phonons from one reservoir to the other happens without reflections. A more realistic model has to incorporate the effects of the reflections caused by the contacts between the reservoirs and the 1D wire. The remainder of this paper is devoted to the study of realistic physical systems in which the universal thermal conductance of phonons could be observed. The systems we consider are similar to the experimental device of Tighe, Worlock and Roukes [8] where a quasi-1D quantum wire connects quasi-2D reservoirs.

We begin our analysis by examining the dispersion relations of low energy phonons in a quasi-2D system. At low temperatures of the order of a few degrees Kelvin, the dominant phonon wavelength is much larger than the lattice parameter and the model of an elastic continuum can be used. We assume an isotropic crystal, which can represent Si or GaAs at low temperatures to a good approximation. The optical phonons are not considered because of their high energy. In this model Rayleigh-Lamb modes [9] describe the quasi-2D acoustic phonons. Figure 1 shows the frequency spectra of the lowest energy symmetric (solid lines) and anti-symmetric (dashed lines) modes of a quasi-2D system of $50 \mathrm{~nm}$ thickness. The parameters used are those of GaAs. The symmetries of the modes refer to atomic displacements in the $z$ direction, perpendicular to the quasi-2D system. The energy scale is degrees Kelvin, so that one may estimate the temperature at which the modes with finite cut-off frequency begin to contribute to thermal transport. These cut-off frequencies increase as the sample thickness is reduced. Together with the $2 \mathrm{D}$ modes of the reservoirs we have plotted in Fig. 1 the lowest energy branches of the longitudinal (circles), transverse (squares), shear (diamonds) and torsional modes (triangles) of a quasi-1D quantum wire with a square cross section of $50 \mathrm{~nm} \times 50 \mathrm{~nm}$. The fundamental torsional mode is given by the simple beam theory, which is well justified by the fact that numerical calculations 10 ] show that its phase velocity is practically constant for all frequencies. The transverse and 
shear modes are coupled by the Timoshenko equation [11]. These quasi-1D modes agree very well at low energies with the $2 \mathrm{D}$ modes of the reservoirs. Therefore they should propagate readily from the reservoirs to the wire. Since expressions (41) and (5) do not depend on the phonon velocity, but only on the cut-off frequencies, the modes shown in Fig. 1 represent the system very well up to temperatures of the order of $1 \mathrm{~K}$.

Nevertheless, there still remains the question of how reflections would change the results given in Eq. (4) and (5). We will discuss this here in detail for the case of the longitudinal modes. For a quasi-1D wire whose cross sectional dimensions are much smaller than its length, the transmitted modes are well described by the normal modes of a long beam. Let us suppose that the cross section of the beam varies along its length, assumed to be in the $x$ direction. The equation of motion of a longitudinal plane wave traveling along the $x$ direction is given by [11]

$$
\frac{\partial^{2} u}{\partial x^{2}}+\frac{1}{A(x)} \frac{\partial A(x)}{\partial x} \frac{\partial u}{\partial x}=\frac{1}{v_{l}^{2}} \frac{\partial^{2} u}{\partial t^{2}} .
$$

$A(x)$ is the cross sectional area of the beam and $v_{l}$ is the velocity of the longitudinal mode $v_{l}=\sqrt{Y / \rho}, Y$ is Young's modulus and $\rho$ the density. To solve Eq.(6) we must specify $A(x)$, so that the transmission coefficients $\zeta(w)$ are determined by the shape of the contacts between the reservoirs and the 1D wire. We consider two contact geometries: conical, for which $A(x)=A_{0} \tan (\theta)\left(x+x^{\prime}\right)$, and catenoidal where $A(x)=A_{0} \cosh ^{2}(x / \lambda)$. These are illustrated in Fig. 2 by schemes (A) and (B), respectively. $\theta$ is the angle of flare of the conical contact and $\lambda$ is the characteristic length of the catenoid. The broad regions at both extremes of the structures are 40 times wider than the 1D channel (50 $\mathrm{nm}$ wide). The thickness (in the direction perpendicular to the plane of the figure, $z$ ) is constant along the whole structure and chosen to be $50 \mathrm{~nm}$. Considering initially the case of conical contacts, two distinct wave equations result for the straight (I) and conical (II) parts of the structure

$$
\begin{aligned}
\frac{\partial^{2} u_{I}}{\partial x^{2}} & =\frac{1}{v_{l}^{2}} \frac{\partial^{2} u_{I}}{\partial t^{2}} \\
\frac{\partial^{2} u_{I I}}{\partial x^{2}}+\frac{1}{x+x^{\prime}} \frac{\partial u_{I I}}{\partial x} & =\frac{1}{v_{l}^{2}} \frac{\partial^{2} u_{I I}}{\partial t^{2}}
\end{aligned}
$$


with solutions

$$
\begin{aligned}
u_{I}(x, t) & =B e^{i( \pm k x-w t)} \\
u_{I I}(x, t) & =C\left[J_{0}\left(k\left(x+x^{\prime}\right)\right) \pm i N_{0}\left(k\left(x+x^{\prime}\right)\right)\right] e^{-i w t} .
\end{aligned}
$$

These solutions are matched at the interfaces between the conical and straight regions, where the continuity of the particle velocities $\dot{u}(x, t)$ and stresses $\sigma=A(x) Y \frac{\partial u(x, t)}{\partial x}$ are required. These boundary conditions lead us to a system of coupled equations that is solved for the transmission coefficients of plane waves traveling along the structure. Fig.2 shows the transmission coefficient $\zeta(k)$ as a function of the longitudinal wave-vector $k$ of a structure with conical contacts in which $\theta=\pi / 6$ (solid line). The peaks are resonances associated with reflections at the ends of the conical regions and of the 1D wire. For $k=0$ the transmission coefficient is equal to one. The overall behavior of $\zeta(k)$ is determined by the conical shape of the contact, that guarantees a finite transmission for all frequencies, but is inefficient for a wide range of frequencies.

The catenoidal contacts present a better response for lower values of $k$. In this case the equations that define the transmitted wave are

$$
\begin{aligned}
\frac{\partial^{2} u_{I}}{\partial x^{2}} & =\frac{1}{v_{l}^{2}} \frac{\partial^{2} u_{I}}{\partial t^{2}} \\
\frac{\partial^{2} u_{I I}}{\partial x^{2}}+\frac{2 \tanh (x / \lambda)}{\lambda} \frac{\partial u_{I I}}{\partial x} & =\frac{1}{v_{l}^{2}} \frac{\partial^{2} u_{I I}}{\partial t^{2}}
\end{aligned}
$$

with solutions

$$
\begin{aligned}
u_{I}(x, t) & =B e^{i( \pm k x-w t)} \\
u_{I I}(x, t) & =C e^{i( \pm k x-w t)}(\cosh (x / \lambda))^{-1} .
\end{aligned}
$$

The transmission coefficients obtained in this case are illustrated by the dashed curve in Fig. 2. Substitution of the function $u_{I I}$ in Eq.(12) results in the frequency spectrum $\omega^{2}=$ $v_{l}^{2}\left(k^{2}+(1 / \lambda)^{2}\right)$ for a wave traveling along the catenoidal contact, which has a cut-off at frequency $\omega_{0}=v_{l} / \lambda$. For $\omega<\omega_{0}$ the wave is evanescent, but a resonance guarantees unitary transmission for $k=0$. However the cut-off frequency becomes smaller as the parameter 
$\lambda$ that characterizes the length of the catenoid increases. The efficiency of transmission of longitudinal waves in this system is also limited by reflections that happen on passing between the straight region and the curved one. These reflections can be avoided for a catenoidal contact that is not laterally limited. This type of contact is exemplified by the structure (C) in Fig. 2, the infinite catenoidal contact. The transmission $\zeta(k)$ for this case is 1 for all wave vectors $k$, but the cut-off at $\omega_{0}=v_{l} / \lambda$ still affects the overall transmission.

With a knowledge of $\zeta(\omega)$ it is possible to obtain realistic results for the 1D thermal conductance. Initially we analyze the case of ideal transmission for all modes, which is a very good approximation for the large $k$ acoustic waves that dominate the thermal transport between $100 \mathrm{mK}$ and $1 \mathrm{~K}$. Six distinct modes contribute to the thermal transport through the wire below 1K: a longitudinal mode, 2 transverse modes, a torsional mode and 2 shear modes. Among these only the shear modes present a cut-off $\left(\hbar \omega_{0}=1.62 K\right)$ that is the result of lateral confinement (see Fig. 四). It was verified that the higher branches of these modes do not contribute significantly to the thermal transport at temperatures lower than $1 \mathrm{~K}$. We considered the limit $\Delta T \rightarrow 0$ of Eq.(5) and calculated the ideal thermal conductance divided by temperature of the quantum wire. The result is shown in Fig. 3 (refer to the left scale). For the ideal contact, at low temperatures, this yields the quantized thermal conductance $k_{B}^{2} \pi^{2} / 3 h$ times the number of massless modes $\left(N_{\alpha}=4\right)$. As the temperature approaches $1 \mathrm{~K}$ the shear modes begin to contribute to the process (increasing the thermal conductance) and the plateau that is the signature of quantized thermal conductance terminates. Now considering the transmission coefficient of the longitudinal mode, we have plotted its contribution to the thermal conductance for various contact shapes (refer to the right scale of Fig. 3). In this case the plateau is modified at the low temperature side by reflections at the contacts and, to a smaller extent, by the cut-off of the frequency spectrum of the catenoidal contacts. Nonetheless for the catenoidal contacts a very distinctive plateau characteristic of quantized thermal conductance is clearly visible over a wide range of temperatures. Therefore based on the behavior of the longitudinal mode, a signature of the quantized thermal conductance of dielectric quantum wires can be expected between $30 \mathrm{mK}$ and $300 \mathrm{mK}$. We 
note that the principle of adiabatic matching that underlies our discussion of the longitudinal mode is very general and applies to the other massless modes as well. Therefore these should exhibit qualitatively similar behavior, including universal quantized thermal conductance over a similar range of temperatures. Since adiabatic wave propagation does not require an isotropic medium, our predictions apply even if there is phonon focusing due to crystal anisotropy. Similar results also apply to wires with cylindrical symmetry which admit analytic solutions for all of the modes, but will be more difficult to fabricate.

In this study we did not consider the effects of surface roughness [8] and other defects that may limit the transmission of phonons through the quantum wire. However these are technological limitations that should be overcome with improved control of the growth process. They should also be of less concern at lower temperatures. [12] Furthermore, studies of $1 \mathrm{D}$ electron transport have shown that quantization plateaus can exist in the presence of defect scattering under certain conditions. [13].

In summary, we predict the existence in $1 \mathrm{D}$ systems of a universal quantum of thermal conductance due to phonons that is equal to $k_{B}^{2} \pi^{2} / 3 h$ times the temperature, for the lowest energy modes. The conductance of the higher energy modes is influenced by the geometrical and intrinsic parameters of the system through the gaps in the frequency spectrum. Realistic structures were investigated and it was found that contacts with catenoidal shape should be those that better represent the ideal case in experiments. Finally, we note that the Wiedemann-Franz law applies in 1D [14], [15] and has been tested experimentally for ballistic point contacts. [16] It predicts [15] that the 1D thermal conductance of ballistic electrons should also be quantized in multiples of $k_{B}^{2} \pi^{2} T / 3 h$. Thus we arrive at the surprising conclusion that the low temperature 1D thermal conductance of ballistic phonons and electrons is described by the same universal quantum even though bosons and fermions obey different statistics.

We thank Michael Roukes for stimulating discussions. This work was supported by NSERC of Canada. 


\section{REFERENCES}

[1] K. von Klitzing, G. Dorda, and M. Pepper, Phys. Rev. Lett. 45, 494 (1980).

[2] B.J. van Wees et al., Phys. Rev. Lett. 60, 848 (1988).

[3] D. A. Wharam et al., J.Phys. C21, L209 (1988)

[4] R. Landauer, IBM J. Res. Dev. 1, 223 (1957); R. Landauer, Phys. Lett. 85A, 91 (1981); L. I. Glazman et al., JETP Lett. 48, 238 (1988); G. Kirczenow, Solid State Commun. 68, 715 (1988); A. Szafer and A. D. Stone, Phys. Rev. Lett. 62, 300 (1989); E. Haanappel and D. van der Marel, Phys. Rev. B 39, 5484 (1989); L. Escapa and N. García, J. Phys. Cond. Matt. 1, 2125 (1989); E. Tekman and S. Ciraçi, Phys. Rev. B39, 8772(1989); Y. Avishai and Y. B. Band, Phys Rev B40, 12535(1989); E. Castaño and G. Kirczenow, Phys. Rev. B45, 1514 (1992).

[5] B. I. Halperin, Phys. Rev. B25, 2185 (1982); M. Büttiker, Phys. Rev. Lett. 57, 1761 (1986); P. Streda, J. Kucera and A. H. MacDonald, Phys. Rev. Lett. 59, 1973 (1987); J. K. Jain and S. A. Kivelson, Phys. Rev. Lett.60, 1542 (1988); M. Büttiker, Phys. Rev. B38, 9375 (1988).

[6] R. E. Peierls, Ann Physik 3, 1055 (1929).

[7] J. Seyler and M.N. Wybourne, Phys. Rev. Lett. 69, 1427 (1992).

[8] T.S. Tighe, J.M. Worlock, and M.L. Roukes, Appl. Phys. Lett. 70, 2687 (1997).

[9] B.A. Auld, Acoustic Fields and Waves in Solids, vol. 2, 2nd edition (Krieger,Malabar,1990).

[10] N. J. Nigro, J. Acous. Soc. Am. 40, 1501 (1966).

[11] K. F. Graff, Wave motion in elastic solids (Ohio State University Press, Columbus,1975).

[12] T. Klitsner et al., Phys. Rev. B 38, 7576 (1988). 
[13] J. A. Nixon and J. H. Davies, Phys. Rev. B 43, 12638 (1991). M. J. Laughton, J. R. Barker, J. A. Nixon, and J. H. Davies, Phys. Rev. B 44, 1150 (1991).

[14] G. V. Chester and A. Thellung, Proc. Phys. Soc. London 77, 1005 (1961); C. Castellani et al., Phys. Rev. Lett.59, 477 (1987).

[15] A. Greiner et al., Phys. Rev. Lett. 78, 1114 (1997).

[16] L.W. Molenkamp et al., Phys. Rev. Lett. 68, 3765 (1992). 


\section{FIGURES}

FIG. 1. Lowest energy acoustic modes of a quasi-2D system of thickness $50 \mathrm{~nm}$ and of a long wire of square cross section $50 \mathrm{~nm} \times 50 \mathrm{~nm}$. Thick solid (dashed) lines represent the symmetric (anti-symmetric) modes of the quasi-2D system. Thin lines with circles, squares, diamonds and triangles represent the longitudinal, transverse, shear and torsional modes of the wire, respectively. The elastic parameters are for GaAs.

FIG. 2. Transmission coefficients vs. longitudinal wave-vector $k$. A) conical contact, $\theta=\pi / 6$; the quasi-1D wire at the center of the structure has cross-section $50 \mathrm{~nm} \times 50 \mathrm{~nm}$ and length $1 \mu$. B) finite catenoidal contact of characteristic length $\lambda=0.86 \mu$. C) infinite catenoidal contact of $\lambda=0.86 \mu$. The straight portions at the ends of structures (A) and (B) have width $2 \mu$.

FIG. 3. Left scale: Thermal conductance of a quantum wire with ideal contacts divided by temperature. Right scale: contribution to thermal conductance due to the longitudinal mode for various contact shapes: infinite catenoid for $\lambda=4.6 \mu$ (solid), finite catenoid for $\lambda=4.6 \mu$ (dot-dashed), catenoid with $\lambda=0.86 \mu$ (long-dashed) and conic for $\theta=\pi / 6$ (solid with circles). $\kappa_{\text {ideal }}^{l}=k_{B}^{2} \pi^{2} T / 3 h$. 